\title{
Systematic Review and Meta-Analysis on Proximal-to-Distal Sequencing in Team Handball: Prospects for Talent Detection?
}

\author{
by \\ Ben Serrien ${ }^{1}$, Jean-Pierre Baeyens ${ }^{1,2,3}$
}

\begin{abstract}
The proximal-to-distal sequence has previously been discussed in the light of performance and injury prevention. Sports biomechanics literature in general, and in team handball in specific, has claimed to be of importance to inform coaches on what constitutes a 'good' technical performance. However, hitherto no prospective studies exist on how this information may be used and this may in part be due to the general small sample sizes. We therefore performed a systematic review and meta-analysis of proximal-to-distal sequencing in team handball throwing motions. A total of fourteen articles were included in the systematic review. Meta-analyses were performed for the timing of maximal angles and angular velocities as well as initiation of joint angular velocities in the penalty throw, the standing throw with run-up and the jump shot of experienced team handball players. For the initiation of joint angular velocities, the overall sequence was estimated to start with pelvis rotation, followed by trunk rotation, trunk flexion, shoulder internal rotation and elbow extension. For maximal velocities, the sequence started with pelvis rotation, followed by trunk rotation, trunk flexion, elbow extension, and shoulder internal rotation (post ball release). The obtained results were discussed in the light of talent identification purposes. Limitations to individual study methodology and of the present meta-analysis were also discussed. Much more research will be necessary, but at the very least, this review can provide a starting point for evidence-based discussions between movement scientists and team handball coaches to include proximal-to-distal sequencing as a measure of coordination to gauge early onset of talent.
\end{abstract}

Key words: team handball, throwing, biomechanics, kinematics, kinetic chain, talent.

\section{Introduction}

Proximal-to-distal sequencing is a phenomenon typical for a large variety of upper limb motions in sports, including baseball pitching (Hirashima et al., 2002), cricket bowling (Ferdinands et al., 2013), javelin throwing (Liu et al., 2010), tennis service, volleyball spiking and team handball throwing (Wagner et al., 2014). The phenomenon is characterized by a progressive sequence of muscle activity and joint motions, starting from the proximal parts of the chain towards the more distal parts. For throwing motions, this indicates a progressive sequence that starts with a thrust of lower limbs, a forward

rotation of the pelvis, followed by the trunk and thereafter the throwing arm. A recent review identified several constraints (torque reversal, biarticular muscles, stretch-shortening cycle, interaction torques, growth, motor development and evolution) likely to contribute to its ubiquity in throwing and striking motions of the upper limb (Serrien and Baeyens, 2017).

Many claims have been made about the relevance of this sequence for sports practitioners in terms of performance enhancement and injury prevention. In baseball throwing, improper timing of trunk rotation has been associated with

\footnotetext{
1 - Vrije Universiteit Brussel, faculty of Physical Education and Physiotherapy, Brussels, Belgium.

2 - Thim Van Der Laan University College Physiotherapy, Landquart, Switzerland.

3 - Universiteit Antwerpen, faculty of Applied Engineering, Antwerp, Belgium.
} 
injury-risk factors such as higher shoulder internal rotation torque (Aguinaldo et al., 2007), higher elbow valgus torque (Aguinaldo and Chambers, 2009) and higher shoulder proximal force and shoulder external rotation angles (Oyama et al., 2014). Also in baseball pitching, Urbin et al. (2013) determined that increased time in the phase from stride-foot-contact to maximalpelvis-rotation and maximal-pelvis-rotation to maximal-trunk-rotation was associated with decreased injury-risk factors (maximal shoulder and elbow joint torques), but at the same time also with decreased ball release speed and that an optimal balance between the two would be important for high-level players.

For team handball throwing, Jöris et al. (1985) were the first to examine this motion pattern and determined a sequence that started with maximal linear hip velocity $(t=128 \mathrm{~ms})$, followed by maximal linear elbow velocity $(t=65$ $\mathrm{ms})$, maximal force exerted on the ball $(\mathrm{t}=31 \mathrm{~ms})$, maximal linear wrist velocity $(\mathrm{t}=24 \mathrm{~ms})$ and maximal energy flow to the ball $(t=23 \mathrm{~ms})$ (all timing variables expressed as mean time before ball release). Subsequent research shifted more towards a description of joint angles and angular velocities. The review article of Wagner et al. (2014) identified the order of maximal joint angular velocities as pelvis rotation, followed by trunk rotation, trunk flexion, elbow extension, shoulder internal rotation, forearm pronation and shoulder flexion.

Research on proximal-to-distal sequencing in team handball has been mainly descriptive with a few quasi-experimental studies that investigated certain constraints such as ball weight (van den Tillaar and Ettema, 2011) and arm dominance (van den Tillaar and Ettema, 2009a). Only limited changes in the timing of joint motions were found. One cross-sectional study addressed the difference in proximal-to-distal sequence between subjects from three skill-levels and determined that mainly for trunk flexion, the timing was significantly earlier and occurred before trunk rotation for the players with the lowest skill level (Wagner et al., 2012a). While gender differences in the time series of various kinematic variables exist (Serrien et al., 2015), the timing seems again to be relatively equal between male and female players (van den Tillaar and Cabri, 2012).
The sequence is not always strictly proximal-to-distal (elbow before shoulder; for a review see Marshall and Elliot, 2000) and task specific adaptations occur. However, overall, the evidence seems to point towards the proximal-todistal sequence being a relatively invariant characteristic of throwing motions in experienced players. Therefore, talent identification programs may potentially benefit from biomechanical analyses of young athletes' throwing patterns. A close fit between pre-existing coordination and control (intrinsic dynamics) and specific task requirements has been proposed to explain precocious talent in sport, where young athletes are able to achieve performance excellence at an early age despite receiving limited coaching and/or engaging in limited practice (Glazier, $2017 \mathrm{~b}$, and the references therein). In addition to other measures of individual performance in team handball (Wagner et al., 2014), the identification of a proximal-to-distal topology in the throwing pattern of young team handball players may be used to gauge talented players. Important to note here, is the notion of coordination variability and the unlikely existence of one 'optimal motion pattern' common to all athletes. We agree with dynamical systems theory of coordination and control (Glazier, 2017a, 2017b; Schöllhorn et al., 2010) that athletes should not be constrained into a highly specific pattern, but that only the topology (shape and timing) of the pattern should be considered relevant. Within a general topology, individual specific optimization may occur.

The aim of this systematic review and meta-analysis was therefore to find a consensus on the timing of joint motions in the proximal-todistal sequence of overhand throwing in experienced team handball players, which could be used to compare junior players' motion patterns. Sports biomechanics studies are often underpowered because of technical and logistical constraints and time-intensive data-processing (Knudson, 2009, 2017). If sports biomechanics research in team handball has the aim to be used for guidance of athletes, meta-analysis of several studies may therefore be a viable option to formulate general guidelines. Team handball has various throwing techniques, but we limited the present analyses to standing throws with and without run-up (penalty throw) and the jump 
throw, which are the most common throws in team handball (Wagner et al., 2008).

\section{Systematic literature search and meta- analysis}

We systematically searched the literature via the following electronic databases: PubMed, SPORTDiscus, Web of Science and Google Scholar. The following keywords were entered in various combinations, using Boolean operators: handball, team handball, throwing, penalty, standing throw with run-up, jump throw, jump shot, proximal-to-distal, sequence, sequencing, kine(ma)tic chain, biomechanics, kinematics, coordination, timing, joint angles, joint angular velocity. Initial screening was performed on titles and abstracts, followed by a profound screening of the full text based on the following inclusion criteria: (1) the subjects were experienced team handball players ( $>5$ years of experience) ('expertise' in sport is a difficult concept and is used heuristically here. See Baker and Young (2014) for a discussion. This cut-off excluded only 1 sample who had less than 2 years of team handball experience); (2) the subjects had no injuries; (3) the study report included data on timing of joint motions (maximal velocity, initiation of velocity, maximal angles) based on three-dimensional motion capturing. No specific limitations were set for the study designs. Further literature search was conducted based on manual searching through the reference lists from included articles. Two additional datasets of our research group were added to the included studies. These datasets contained time series of joint angles and angular velocities and were published before (Serrien et al., 2015, 2016), but did not include explicit data on timing of maximal joint motions. They were thus re-analyzed to compute the data on timing of maximal joint velocity and angles and timing of initiation of velocity.

The following seven degrees-of-freedom were selected for inclusion: pelvis rotation, trunk rotation, trunk flexion, shoulder horizontal ab/adduction, shoulder internal rotation, elbow flexion/extension, wrist flexion/extension. Initial screening showed that these variables were reported frequently enough to perform a metaanalysis and allowed to sketch a complete picture of the proximal-to-distal sequence. Following the arguments in the introduction that only the global topology is relevant for identifying coordination, we chose not to include every degree-of-freedom that was reported in literature. When the article contained only figures, the authors were contacted by email for the exact data (means and $\mathrm{SDs}$ ) to perform the meta-analysis. Open source software from the Cochrane Collaboration (Review Manager 5.3) was used to conduct the meta-analysis. For each study (subgroup, time point), the mean and standard error $(\mathrm{SD} / \mathrm{V} \mathrm{n})$ for each variable were entered to estimate an overall $95 \%$ CI. The inverse variance method was used to weight the influence of each study and the random effects model was used in each metaanalysis (Borenstein et al., 2009). We calculated 95\% prediction intervals (PI) in Excel using the $\mathrm{T}^{2}$ estimate and formulas from Borenstein et al. (2009, chapter 17). Confidence intervals give only a spread on the mean timing, while prediction intervals are able to predict the range of future sample means, taking the between-study variance into account (the intervals are equal when $\mathrm{T}^{2}=\mathrm{I}^{2}=$ $0)$. Heterogeneity between the studies was reported as $\mathrm{I}^{2}$, which reflects the extent of overlap between the $\mathrm{CI}$ of several studies (Borenstein et al., 2009). A minimum of five estimates, from independent samples, from minimal two research groups was deemed necessary to perform a meaningful meta-analysis.

Table 1 presents the design of each included study and the subject characteristics. A total of fourteen articles were included in the review and meta-analysis. The main reason for exclusion of an article was that it contained only data on the values of angular velocities and not their timing (Wagner and Müller, 2008) or that it contained only timing of linear velocities (Jöris et al., 1985; van den Tillaar and Ettema, 2003, 2006). Articles that contained data on independent subgroups (gender, skill level) were entered separately in the meta-analyses if the subgroups met the inclusion criteria. For the study that compared different ball weights, only the data for the regular ball weight was taken into account (van den Tillaar and Ettema, 2011). For the study that compared throwing with both arms, only the data from the dominant arm was taken into the meta-analysis (van den Tillaar and Ettema, 2009a). For the studies with multiple conditions/time points, all data were used in the meta-analyses as 
these concerned natural conditions within a throwing type and thus relevant for identification of a global topology: circular and whip-like windups, arm position above/side, pre/post training, intra-seasonal follow up (Serrien et al., 2016; van den Tillaar and Marques, 2011; van den Tillaar et al., 2013; Wagner et al., 2010a). However, because these data were not independent and would thus overestimate the accuracy of the metaanalysis, the multiple conditions/time points were concatenated into one estimate per study (Borenstein et al., 2009, chapter 24). Since the correlations were unknown in most studies, we used an ad-hoc estimate of $r=0.50$, based on the data of Serrien et al. (2016) where the correlations between the three time points in the study were $0.56,0.54$ and 0.59 (see the discussion for implications of this estimate).

\section{Results}

Figure 1 shows the forest plot of one meta-analysis (timing of maximal elbow extension velocity in the penalty throw). The heterogeneity between samples $(\mathrm{n}=10)$ was relatively large $\left(\mathrm{I}^{2}=\right.$ $87 \%)$. The overall mean was estimated as $8.51 \mathrm{~ms}$ prior to ball release (95\% CI: [-10.66 to -6.37] ms). The 95\% PI was calculated in Excel: [-15.4 -1.6] ms.

The same analysis was performed for each variable and the results are shown in Table 2 (timing of maximal angles) and Table 3 (timing of angular velocity initiation) for the penalty throw. Note that data for studies with multiple conditions/time points are shown separately, yet they were combined in one estimate for conduction of the meta-analysis. Since data on timing of wrist motions were scarce, a metaanalysis for its timing was not performed, but raw study data are shown. Table 4 shows the metaanalyses on timing of maximal joint velocity for the penalty throw, the standing throw with runup and the jump shot. Shoulder horizontal ab/adduction was eventually not included because of methodological differences between studies (see notes in the discussion).

\section{Discussion}

The meta-analyses performed in this study aimed at finding a consensus on the general topology of the proximal-to-distal sequence in team handball throwing. The included studies were relatively homogeneous in sample characteristics (age, body height, body mass, team handball experience). Only the competition level showed more heterogeneity, even within certain samples, ranging from (youth) national teams to $3^{\text {rd }}$ and $4^{\text {th }}$ national competition levels.

For the initiation of joint angular velocities in the penalty throw, the overall sequence was estimated to start with pelvis rotation, followed by trunk rotation, trunk flexion, shoulder internal rotation and elbow extension. For maximal velocities (see Figure 2 for a synthesis), the sequence started with pelvis rotation, followed by trunk rotation, trunk flexion, elbow extension, and shoulder internal rotation (post ball release). The same order occurred for the maximal velocities in the penalty throw, the standing throw with run-up and jump throws and for some degrees-of-freedom, even the absolute timing was nearly identical. For instance, maximal elbow extension occurred on average 9, 11 and 13 ms prior to ball release for the penalty throw, the standing throw with run-up and the jump throw, respectively, which are all located within the temporal resolution of the sampling frequency (typical $250 \mathrm{~Hz}, 4 \mathrm{~ms}$ resolution). The results of the meta-analysis confirm the observation of van den Tillaar and Ettema (2009b) that initiation of angular velocities follows a strict proximal-todistal sequence, while for maximal velocities, order of the shoulder and elbow is reversed. Before we continue with a discussion on the possible applications of these results, we will consider a few methodological issues and limitations of the meta-analysis.

\section{Methodological issues}

In most variables, the heterogeneity between studies $\left(\mathrm{I}^{2}\right)$ was large which may be partially explained by differences in methodologies. The experimental procedures and data recording were relatively homogeneous between the different studies, but the kinematic analyses showed important differences. Especially when future studies may attempt to perform meta-analyses on measures of coordination that are more sensitive, like time series data (Serrien et al., 2015), phase plane analysis (Wagner et al., $2012 b)$ or coordination profiling with neural networks (Serrien et al., 2016), a good and consistent definition of variables will be important for meaningful comparisons between studies. 


\section{Table 1}

Study sample characteristics (means \pm SD) for the included articles.

\begin{tabular}{|c|c|c|c|c|c|c|c|}
\hline $\begin{array}{l}\text { First author, } \\
\text { year } \\
\text { Study ID }\end{array}$ & $\begin{array}{c}\text { Desig } \\
\mathrm{n}\end{array}$ & $\mathrm{N}$, gender & Age (yrs.) & $\begin{array}{l}\text { Body } \\
\text { height } \\
(\mathrm{cm})\end{array}$ & $\begin{array}{c}\text { Body } \\
\text { mass }(\mathrm{kg})\end{array}$ & $\begin{array}{c}\text { Handball } \\
\text { experienc } \\
\text { e (yrs.) }\end{array}$ & Competitive level \\
\hline $\begin{array}{l}\text { van den } \\
\text { Tillaar, } 2007\end{array}$ & CS & 11 , male & $22.9 \pm 3.5$ & $\begin{array}{c}184 \pm \\
5\end{array}$ & $85.8 \pm 11.8$ & $13 \pm 3.3$ & $\begin{array}{l}\text { Top and first division of the } \\
\text { Norwegian national competition }\end{array}$ \\
\hline $\begin{array}{l}\text { van den } \\
\text { Tillaar, 2009a }\end{array}$ & CS & 11 , male & $22.9 \pm 3.5$ & $\begin{array}{c}184 \pm \\
5\end{array}$ & $85.8 \pm 11.8$ & $13 \pm 3.3$ & $\begin{array}{l}\text { Top and first division of the } \\
\text { Norwegian national competition }\end{array}$ \\
\hline $\begin{array}{l}\text { van den } \\
\text { Tillaar, } 2009 b^{c}\end{array}$ & QE & 11 , male & $22.9 \pm 3.5$ & $\begin{array}{c}184 \pm \\
5\end{array}$ & $85.8 \pm 11.8$ & $13 \pm 3.3$ & $\begin{array}{l}\text { Top and first division of the } \\
\text { Norwegian national competition }\end{array}$ \\
\hline Wagner, 2010a & CS & 12 , male & $22.5 \pm 3.7$ & $\begin{array}{c}187 \pm \\
6\end{array}$ & $84.4 \pm 10.5$ & $10.3 \pm 3.6$ & $\begin{array}{c}1^{\text {st }} 3^{\text {rd }} \text { Austrian, } 1^{\text {st }} \text { Spanish and } \\
\text { German competitions }\end{array}$ \\
\hline Wagner, 2010b & CS & 12 , male & $22.5 \pm 3.7$ & $\begin{array}{c}187 \pm \\
6\end{array}$ & $84.4 \pm 10.5$ & $11.6 \pm 3.2$ & $\begin{array}{l}\text { Austrian national team, } 2^{\text {nd }} 3^{\text {rd }} \\
\text { Austrian/German competition }\end{array}$ \\
\hline $\begin{array}{l}\text { van den Tillaar } \\
\text { and Ettema, } \\
2011^{c}\end{array}$ & QE & 24 , female & $18.2 \pm 2.1$ & $\begin{array}{c}169 \pm \\
4\end{array}$ & $68.0 \pm 7.0$ & $10.7 \pm 1.9$ & $\begin{array}{l}2^{\text {nd }} \text { and } 3^{\text {rd }} \text { division of the } \\
\text { Norwegian national competition }\end{array}$ \\
\hline $\begin{array}{l}\text { van den Tillaar } \\
\text { and Marques, } \\
2011^{b}\end{array}$ & QE & 9 , female & $19.9 \pm 2.1$ & $\begin{array}{c}169 \pm \\
3\end{array}$ & $67.3 \pm 7.5$ & $11.0 \pm 1.5$ & $\begin{array}{c}2^{\text {nd }}-4^{\text {th }} \text { division of the Norwegian } \\
\text { national competition }\end{array}$ \\
\hline Wagner, 2011 & CS & 14 , male & $22.9 \pm 4.2$ & $\begin{array}{c}185 \pm \\
7\end{array}$ & $82.4 \pm 11.1$ & $10.8 \pm 3.8$ & $\begin{array}{c}1^{\text {st }} 3^{\text {rd }} \text { Austrian, } 1^{\text {st }} \text { Spanish and } \\
\text { German competitions }\end{array}$ \\
\hline Wagner, 2012a & CS & 10 , male & $23.0 \pm 3.0$ & $\begin{array}{c}187 \pm \\
6\end{array}$ & $85.0 \pm 10.0$ & $12.0 \pm 3.0$ & $\begin{array}{c}\text { Austrian national team, } 2^{\text {nd }} \text { and } \\
3^{\text {rd }} \text { Austrian competition }\end{array}$ \\
\hline Wagner, 2014a & CS & 8 , male & $19.1 \pm 3.1$ & $\begin{array}{c}184 \pm \\
5\end{array}$ & $76.4 \pm 8.4$ & $6.6 \pm 2.0$ & Austrian regional handball team \\
\hline \multirow{3}{*}{$\begin{array}{l}\text { van den } \\
\text { Tillaar, 2012a }\end{array}$} & \multirow{3}{*}{ CS } & 8 , male & $25.3 \pm 3.2$ & $\begin{array}{c}185 \pm \\
9\end{array}$ & $85.6 \pm 12.0$ & $13.4 \pm 2.1$ & $\begin{array}{l}\text { Austrian national team and } 2^{\text {nd }} \\
\text { Austrian national competition }\end{array}$ \\
\hline & & 11 , male & $23.6 \pm 5.2$ & $\begin{array}{c}185 \pm \\
5\end{array}$ & $87.0 \pm 6.8$ & $12.7 \pm 3.8$ & $\begin{array}{l}\text { Elite players in the Norwegian } \\
\text { national competition }\end{array}$ \\
\hline & & 11 , female & $20.3 \pm 1.8$ & $\begin{array}{c}175 \pm \\
5\end{array}$ & $69.9 \pm 5.5$ & $12.8 \pm 2.0$ & $\begin{array}{l}\text { Elite players in the Norwegian } \\
\text { national competition }\end{array}$ \\
\hline \multirow[t]{2}{*}{$\begin{array}{l}\text { van den } \\
\text { Tillaar, } 2013\end{array}$} & \multirow[t]{2}{*}{ QE } & 11 , male & $23.6 \pm 5.2$ & $\begin{array}{c}185 \pm \\
5\end{array}$ & $87.0 \pm 6.8$ & $12.7 \pm 3.8$ & $\begin{array}{l}\text { Elite players in the Norwegian } \\
\text { national competition }\end{array}$ \\
\hline & & 11 , female & $20.3 \pm 1.8$ & $\begin{array}{c}175 \pm \\
5\end{array}$ & $69.9 \pm 5.5$ & $12.8 \pm 2.0$ & $\begin{array}{l}\text { Elite players in the Norwegian } \\
\text { national competition }\end{array}$ \\
\hline \multirow[t]{2}{*}{ Serrien, 2015 } & \multirow[t]{2}{*}{ CS } & 10 , male & $25.4 \pm 4.0$ & $\begin{array}{c}182 \pm \\
5\end{array}$ & $86.2 \pm 12.5$ & $11.4 \pm 4.7$ & $\begin{array}{l}2^{\text {nd }} \text { division of the Swiss national } \\
\text { competition }\end{array}$ \\
\hline & & 10 , female & $23.7 \pm 2.7$ & $\begin{array}{c}169 \pm \\
6\end{array}$ & $63.7 \pm 4.7$ & $13.1 \pm 4.1$ & $\begin{array}{l}2^{\text {nd }} \text { division of the Swiss national } \\
\text { competition }\end{array}$ \\
\hline Serrien, 2016 b & LC & 13 , male & $18.4 \pm 0.5$ & $\begin{array}{c}178 \pm \\
8\end{array}$ & $73.6 \pm 9.4$ & $9.0 \pm 2.4$ & $\begin{array}{l}\text { U19 Belgian national team, 1 }{ }^{\text {st- }} \\
2^{\text {nd }} \text { Belgian competition }\end{array}$ \\
\hline
\end{tabular}

Abbreviations: $C S=$ cross-sectional, $Q E=$ quasi-experimental, $L C=$ longitudinal cohort. Notes: 'subgroups from this study were treated as independent samples for the meta-analysis; ${ }^{b}$ multiple time points were combined in one estimate for the meta-analysis; conly one condition of the task was used for the meta-analysis. 


\begin{tabular}{|c|c|c|c|c|c|c|}
\hline \multicolumn{7}{|c|}{$\begin{array}{l}\text { Timing of maximal angles (penalty throw). Mean } \pm \text { SE per study sample/condition/time point } \\
\text { and results of the meta-analysis (milliseconds prior(-) or post (+) ball release). }\end{array}$} \\
\hline Study ID & $\begin{array}{l}\text { Pelvis } \\
\text { rotation }\end{array}$ & $\begin{array}{c}\text { Trunk } \\
\text { rotation }\end{array}$ & $\begin{array}{c}\text { Trunk } \\
\text { extension }\end{array}$ & $\begin{array}{c}\text { Shoulder } \\
\text { external } \\
\text { rotation }\end{array}$ & Elbow flexion & $\begin{array}{c}\text { Wrist } \\
\text { extension }\end{array}$ \\
\hline \multicolumn{7}{|l|}{ PENALTY THROW } \\
\hline van den Tillaar, 2007 & $-474 \pm 46.2$ & $-304 \pm 25.0$ & $-299 \pm 60.4$ & $-70 \pm 4.4$ & $-64 \pm 3.2$ & $-373 \pm 55.0$ \\
\hline van den Tillaar, 2009b & $-270 \pm 22.9$ & $-241 \pm 17.5$ & $-221 \pm 25.6$ & $-65 \pm 3.6$ & $-102 \pm 16.3$ & $-187 \pm 17.5$ \\
\hline van den Tillaar, 2011a & $-415 \pm 19.4$ & $-280 \pm 9.4$ & $-389 \pm 12.7$ & $-105 \pm 4.7$ & $-63 \pm 2.4$ & - \\
\hline van den Tillaar, 2011b (pre) & $-398 \pm 42.7$ & $-296 \pm 22.0$ & $-322 \pm 29.3$ & $-167 \pm 19.7$ & $-73 \pm 11.7$ & - \\
\hline van den Tillaar, 2011b (post) & $-389 \pm 29.7$ & $-283 \pm 20.0$ & $-314 \pm 60.3$ & $-182 \pm 29.3$ & $-60 \pm 3.7$ & - \\
\hline van den Tillaar, 2012 (male) & $-383 \pm 23.8$ & $-280 \pm 10.6$ & $-372 \pm 30.5$ & $-125 \pm 11.2$ & $-898 \pm 202.6$ & $-19 \pm 2.4$ \\
\hline $\begin{array}{l}\text { van den Tillaar, } 2012 \\
\text { (female) }\end{array}$ & $-393 \pm 20.2$ & $-275 \pm 12.1$ & $-310 \pm 19.9$ & $-135 \pm 16.3$ & $-1161 \pm 199.9$ & $-18 \pm 1.5$ \\
\hline $\begin{array}{l}\text { van den Tillaar, } 2013 \\
\text { (circular) }\end{array}$ & $-508 \pm 38.6$ & $-278 \pm 7.9$ & $-440 \pm 19.2$ & $-80 \pm 2.8$ & $-556 \pm 59.7$ & - \\
\hline $\begin{array}{l}\text { van den Tillaar, } 2013 \\
\text { (whip) }\end{array}$ & $-516 \pm 43.3$ & $-276 \pm 6.4$ & $-473 \pm 24.7$ & $-94 \pm 4.3$ & $-397 \pm 42.6$ & - \\
\hline Serrien, 2016 (February) & $-376 \pm 12.9$ & $-278 \pm 8.0$ & $-276 \pm 6.3$ & $-103 \pm 3.1$ & $-88 \pm 2.1$ & - \\
\hline Serrien, 2016 (April) & $-389 \pm 23.1$ & $-287 \pm 8.1$ & $-275 \pm 8.1$ & $-107 \pm 3.0$ & $-85 \pm 3.7$ & - \\
\hline Serrien, 2016 (May) & $-381 \pm 19.8$ & $-293 \pm 9.0$ & $-298 \pm 12.9$ & $-106 \pm 3.2$ & $-84 \pm 3.9$ & - \\
\hline MEAN & -399 & -280 & -333 & -102 & -95 & \\
\hline $95 \%$ CI & {$[-430-369]$} & {$[-286-275]$} & {$[-371-296]$} & [-114 -91] & {$[-110-80]$} & \\
\hline $95 \%$ PI & {$[-456-342]$} & {$[-287-274]$} & {$\left[\begin{array}{ll}-389 & -278\end{array}\right]$} & {$[-121-85]$} & {$[-122-68]$} & \\
\hline Heterogeneity $\left(\mathrm{I}^{2}\right)$ & $79 \%$ & $0 \%$ & $94 \%$ & $95 \%$ & $96 \%$ & \\
\hline
\end{tabular}

Table 3

Timing of angular velocity initiation (penalty throw). Mean \pm SE per study sample/condition/time point and results of the meta-analysis (milliseconds prior(-) or post (+) ball release).

\begin{tabular}{|c|c|c|c|c|c|c|}
\hline Study ID & $\begin{array}{l}\text { Pelvis } \\
\text { rotation }\end{array}$ & $\begin{array}{l}\text { Trunk } \\
\text { rotation }\end{array}$ & $\begin{array}{l}\text { Trunk } \\
\text { flexion }\end{array}$ & $\begin{array}{l}\text { Shoulder internal } \\
\text { rotation }\end{array}$ & $\begin{array}{l}\text { Elbow } \\
\text { extension }\end{array}$ & $\begin{array}{l}\text { Wrist } \\
\text { flexion }\end{array}$ \\
\hline \multicolumn{7}{|l|}{ PENALTY THROW } \\
\hline van den Tillaar, 2009a & $-418 \pm 36.5$ & $-247 \pm 22.0$ & $-206 \pm 22.6$ & $-71 \pm 4.2$ & $-65 \pm 3.3$ & $-35 \pm 6.6$ \\
\hline van den Tillaar, 2012 (male) & $-297 \pm 49.4$ & $-282 \pm 9.6$ & $-275 \pm 28.0$ & $-111 \pm 10.6$ & $-69 \pm 4.2$ & $-21 \pm 2.1$ \\
\hline $\begin{array}{l}\text { van den Tillaar, } 2012 \\
\text { (female) }\end{array}$ & $-229 \pm 51.6$ & $-267 \pm 7.5$ & $-259 \pm 25.0$ & $-129 \pm 16.3$ & $-67 \pm 2.4$ & $-20 \pm 1.2$ \\
\hline $\begin{array}{l}\text { van den Tillaar, } 2013 \\
\text { (circular) }\end{array}$ & $-353 \pm 15.6$ & $-274 \pm 6.2$ & $-353 \pm 22.4$ & $-80 \pm 2.8$ & $-68 \pm 2.3$ & - \\
\hline van den Tillaar, 2013 (whip) & $-355 \pm 15.6$ & $-263 \pm 13.0$ & $-320 \pm 28.1$ & $-94 \pm 4.3$ & $-75 \pm 3.2$ & - \\
\hline Serrien, 2016 (February) & $-312 \pm 12.4$ & $-251 \pm 12.3$ & $-199 \pm 13.1$ & $-102 \pm 8.1$ & $-71 \pm 4.1$ & - \\
\hline Serrien, 2016 (April) & $-309 \pm 13.2$ & $-262 \pm 15.0$ & $-192 \pm 12.1$ & $-100 \pm 10.2$ & $-64 \pm 2.9$ & - \\
\hline Serrien, 2016 (May) & $-321 \pm 12.1$ & $-259 \pm 11.1$ & $-197 \pm 14.4$ & $-96 \pm 2.9$ & $-68 \pm 3.1$ & - \\
\hline MEAN & -330 & -268 & -247 & -94 & -68 & \\
\hline $95 \%$ CI & {$\left[\begin{array}{lll}-352 & -308\end{array}\right]$} & [-275 -261] & {$\left[\begin{array}{ll}-286 & -208\end{array}\right]$} & {$[-103-84]$} & {$[-70-66]$} & \\
\hline $95 \%$ PI & [-362 -299] & {$[-276-259]$} & {$\left[\begin{array}{ll}-290 & -204\end{array}\right]$} & {$\left[\begin{array}{ll}-112 & -76\end{array}\right]$} & {$[-74-62]$} & \\
\hline Heterogeneity (I²) & $65 \%$ & $0 \%$ & $89 \%$ & $86 \%$ & $15 \%$ & \\
\hline
\end{tabular}


Table 4

Timing of maximal velocities (penalty throw, standing throw with run-up and jump shot).

Mean \pm SE per study sample/condition/time point and results of the meta-analysis (milliseconds prior(-) or post $(+)$ ball release).

\begin{tabular}{|c|c|c|c|c|c|c|}
\hline Study ID & $\begin{array}{c}\text { Pelvis } \\
\text { rotation }\end{array}$ & $\begin{array}{c}\text { Trunk } \\
\text { rotation }\end{array}$ & $\begin{array}{l}\text { Trunk } \\
\text { flexion }\end{array}$ & $\begin{array}{c}\text { Shoulder internal } \\
\text { rotation* }\end{array}$ & $\begin{array}{c}\text { Elbow } \\
\text { extension }\end{array}$ & $\begin{array}{l}\text { Wrist } \\
\text { flexion }\end{array}$ \\
\hline \multicolumn{7}{|l|}{ PENALTY THROW } \\
\hline van den Tillaar, 2009a & $-103 \pm 9.0$ & $-49 \pm 3.0$ & $-20 \pm 3.6$ & $21 \pm 2.4$ & $-11 \pm 2.4$ & $-18 \pm 3.3$ \\
\hline van den Tillaar, $2009 b$ & $-112 \pm 12.7$ & $-53 \pm 3.0$ & $-37 \pm 13.3$ & - & $-17 \pm 6.0$ & $-37 \pm 1.2$ \\
\hline van den Tillaar, 2011a & $-108 \pm 4.7$ & $-52 \pm 4.7$ & $-20 \pm 3.1$ & - & $-5 \pm 1.2$ & - \\
\hline van den Tillaar, $2011 b$ (pre) & $-110 \pm 9.0$ & $-65 \pm 9.7$ & $-23 \pm 4.7$ & - & $-7 \pm 2.0$ & - \\
\hline $\begin{array}{l}\text { van den Tillaar, } 2011 b \\
\text { (post) }\end{array}$ & $-105 \pm 8.3$ & $-53 \pm 9.3$ & $-19 \pm 4.3$ & - & $-4 \pm 1.7$ & - \\
\hline Wagner, 2011 & $-112 \pm 6.1$ & $-82 \pm 5.3$ & - & $4 \pm 1.1$ & $-14 \pm 1.1$ & - \\
\hline van den Tillaar, 2012 (male) & $-108 \pm 11.5$ & $-58 \pm 5.4$ & $-35 \pm 4.8$ & - & $-7 \pm 1.5$ & $-1 \pm 0.9$ \\
\hline $\begin{array}{l}\text { van den Tillaar, } 2012 \\
\text { (female) }\end{array}$ & $-113 \pm 7.8$ & $-59 \pm 4.5$ & $-40 \pm 4.2$ & - & $-8 \pm 1.8$ & $0 \pm 0.1$ \\
\hline $\begin{array}{l}\text { van den Tillaar, } 2013 \\
\text { (circular) }\end{array}$ & $-134 \pm 4.3$ & $-58 \pm 3.4$ & $-54 \pm 2.8$ & - & $-10 \pm 1.1$ & - \\
\hline $\begin{array}{l}\text { van den Tillaar, } 2013 \\
\text { (whip) }\end{array}$ & $-161 \pm 7.7$ & $-61 \pm 4.7$ & $-55 \pm 3.0$ & - & $-8 \pm 1.3$ & - \\
\hline Serrien, 2016 (February) & $-117 \pm 5.8$ & $-56 \pm 2.8$ & $-38 \pm 3.3$ & $4 \pm 0.6$ & $-8 \pm 0.8$ & - \\
\hline Serrien, 2016 (April) & $-112 \pm 3.3$ & $-52 \pm 2.5$ & $-39 \pm 3.6$ & $3 \pm 0.6$ & $-4 \pm 0.6$ & - \\
\hline Serrien, 2016 (May) & $-125 \pm 6.7$ & $-62 \pm 4.2$ & $-44 \pm 4.2$ & $4 \pm 0.4$ & $-4 \pm 1.4$ & - \\
\hline MEAN & -118 & -57 & -34 & 6 & -9 & \\
\hline $95 \%$ CI & [-125 -110] & {$\left[\begin{array}{ll}-61 & -53]\end{array}\right.$} & {$\left[\begin{array}{ll}-42 & -26\end{array}\right]$} & [4 8] & {$[-11-6]$} & \\
\hline $95 \%$ PI & {$[-146-90]$} & {$\left[\begin{array}{ll}-66 & -49]\end{array}\right.$} & {$[-47-21]$} & [0 12] & {$[-15-2]$} & \\
\hline Heterogeneity $\left(\mathrm{I}^{2}\right)$ & $79 \%$ & $69 \%$ & $93 \%$ & $91 \%$ & $87 \%$ & \\
\hline \multicolumn{7}{|c|}{ STANDING THROW WITH RUN-UP } \\
\hline Wagner, 2010a (arm above) & - & $-83 \pm 5.2$ & $-47 \pm 4.6$ & $15 \pm 1.7$ & $-14 \pm 3.5$ & - \\
\hline Wagner, 2010a (arm side) & - & $-91 \pm 5.5$ & $-2 \pm 3.5$ & $19 \pm 3.5$ & $-12 \pm 3.5$ & - \\
\hline Wagner, 2011 & $-110 \pm 7.2$ & $-80 \pm 4.8$ & - & $4 \pm 1.3$ & $-14 \pm 1.1$ & - \\
\hline $\begin{array}{l}\text { Wagner, 2012a } \\
\text { (experienced) }\end{array}$ & $-107 \pm 7.8$ & $-92 \pm 5.7$ & $-77 \pm 13.4$ & $9 \pm 2.1$ & $-10 \pm 1.8$ & $28 \pm 4.2$ \\
\hline Wagner, 2012a (elite) & $-106 \pm 11$ & $-75 \pm 6.4$ & $-44 \pm 4.2$ & $3 \pm 1.8$ & $-15 \pm 1.4$ & $28 \pm 2.5$ \\
\hline Serrien, 2015 (male) & $-107 \pm 7$ & $-83 \pm 3.8$ & $-67 \pm 6.6$ & $8 \pm 2.9$ & $-4 \pm 2.1$ & - \\
\hline Serrien, 2015 (female) & $-109 \pm 5.7$ & $-76 \pm 4.1$ & $-63 \pm 8.9$ & $10 \pm 2.0$ & $-8 \pm 2.0$ & - \\
\hline MEAN & -108 & -84 & -49 & 9 & -11 & \\
\hline $95 \%$ CI & {$[-115-102]$} & {$\left[\begin{array}{ll}-90 & -79]\end{array}\right.$} & {$[-73-25]$} & {$\left[\begin{array}{ll}5 & 13\end{array}\right]$} & {$[-14-8]$} & \\
\hline $95 \%$ PI & {$[-116-101]$} & {$[-93-76]$} & {$\left[\begin{array}{ll}-82 & -14\end{array}\right]$} & [1 17] & {$[-22-1]$} & \\
\hline Heterogeneity $\left(\mathrm{I}^{2}\right)$ & $0 \%$ & $62 \%$ & $97 \%$ & $87 \%$ & $79 \%$ & \\
\hline \multicolumn{7}{|l|}{ JUMP THROW } \\
\hline Wagner, 2010b & $-130 \pm 11.5$ & $-87 \pm 5.2$ & $-51 \pm 5.8$ & $3 \pm 0.8$ & $-18 \pm 4.0$ & $0 \pm 9.5$ \\
\hline Wagner, 2011 & $-122 \pm 6.9$ & $-90 \pm 5.1$ & - & $4 \pm 1.3$ & $-17 \pm 3.5$ & - \\
\hline Wagner, 2012a & $-118 \pm 5.7$ & $-89 \pm 5.4$ & $-47 \pm 4.1$ & $3 \pm 0.9$ & $-20 \pm 5.7$ & - \\
\hline Serrien, 2016 (February) & $-121 \pm 3.9$ & $-82 \pm 4.2$ & $-47 \pm 3.8$ & $8 \pm 1.2$ & $-12 \pm 3.1$ & - \\
\hline Serrien, 2016 (April) & $-129 \pm 4.1$ & $-75 \pm 3.9$ & $-52 \pm 3.1$ & $7 \pm 1.8$ & $-11 \pm 1.8$ & - \\
\hline Serrien, 2016 (May) & $-119 \pm 4.0$ & $-84 \pm 3.1$ & $-55 \pm 3.9$ & $7 \pm 1.5$ & $-8 \pm 2.2$ & - \\
\hline MEAN & -122 & -84 & -51 & 5 & -13 & \\
\hline $95 \%$ CI & {$\left[\begin{array}{ll}-126 & -118\end{array}\right]$} & {$\left[\begin{array}{ll}-88 & -79]\end{array}\right.$} & {$[-54-47]$} & [3 7] & [-16 -9] & \\
\hline $95 \%$ PI & {$\left[\begin{array}{lll}-127 & -117]\end{array}\right]$} & {$\left[\begin{array}{ll}-99 & -68\end{array}\right]$} & {$[-55-46]$} & {$\left[\begin{array}{ll}-1 & 11\end{array}\right]$} & {$\left[\begin{array}{ll}-20 & -5\end{array}\right]$} & \\
\hline Heterogeneity (I²) & $0 \%$ & $38 \%$ & $0 \%$ & $75 \%$ & $49 \%$ & \\
\hline
\end{tabular}

* note: some studies did not include data post ball release and maximal shoulder internal rotation could not be estimated 

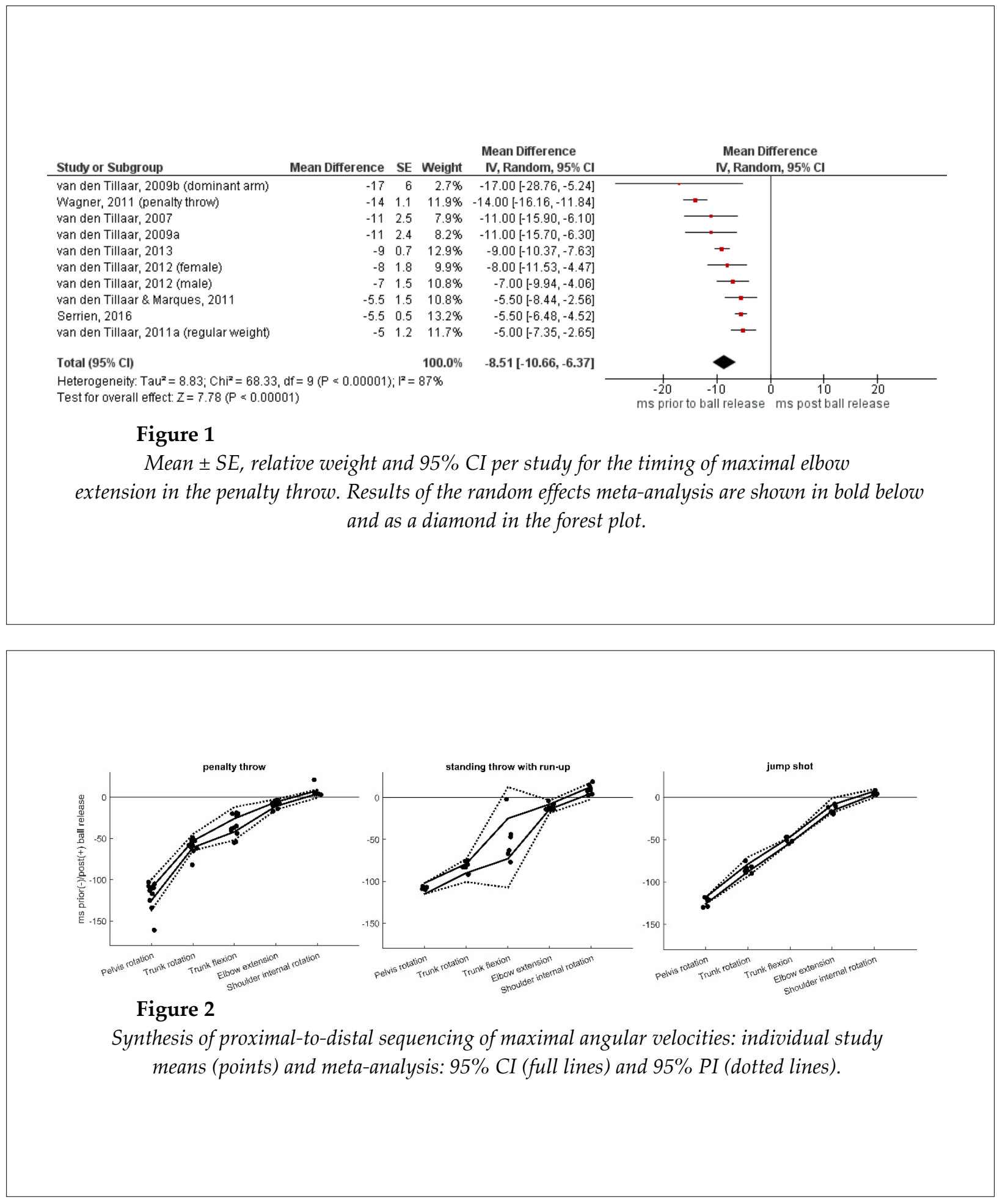

The articles from van den Tillaar et al. employed the methods published by Feltner and Dapena (1989), Wagner et al. used the VICON Plug-in-Gait model and Serrien et al. used the ISB guidelines (Wu et al., 2002, 2005) for calculations of joint kinematics. While all three methods are valid in se, they may strongly disagree when compared to each other, especially in shoulder kinematics (Baeyens et al., 2000, 2001; BonnefoyMazure et al., 2010; Creveaux et al., 2017). For instance, the VICON Plug-in-Gait model uses a YXZ rotation sequence of shoulder flexion/extension, ab/adduction, internal/external rotation, whereas the ISB guidelines specify a YXY 
rotation sequence of the shoulder plane of elevation (horizontal ab/adduction), ab/adduction, internal/external rotation. Because of these differences, we took only shoulder internal/external rotation into account in the metaanalyses.

With regard to the time span of interest, important for future studies on shoulder rotation is that data post ball release are taken into account as many studies were not able to estimate the occurrence of the true maximum of this variable. On average, the time span of interest should cover between -500 to $+50 \mathrm{~ms}$ to be able to estimate the timing of initiation and timing of maximal velocities of all variables of interest. Based on the few estimates of maximal wrist flexion velocity, this could also be covered in this time frame. Another degree-of-freedom which has its maximal velocity post ball release on average is elbow pronation. Only two studies provided data on this variable (Wagner et al., 2010b, 2012a) and was therefore not included. However, it may be relevant to include it as a measure of an efficient follow-through. As Zhang et al. (2018) showed in their experiments with throwing tasks, creating a window for ball release that is tolerant to neuromuscular noise requires shaping the arm trajectory before and after release and thus coaches should pay attention to it.

A final methodological issue is the coverage of only one level of analysis in team handball literature, namely the kinematic level. Especially for theoretical motor control purposes, it would be important to establish how the proximal-to-distal sequence is presented at the level of joint kinetics and muscle activity (sEMG) in order to examine how this topology at one level is constrained by the dynamics of another level (Serrien and Baeyens, 2017).

\section{Limitations of the meta-analysis}

The most important limitation concerns the low number of included studies, which came from only three research groups. Each of the studies had only low sample sizes $(<25$, Table 1$)$ and not enough data were available to warrant further subgroup analyses (e.g. male vs. female players). Also the between-sample variability in team handball experience and especially in competitive levels is a limitation and warrants further subgroup analyses. As may be seen in Table 1, some studies employed the same subjects, which does not increase the overall sample size. These studies were taken into the meta-analysis as independent because they were published separately, but this limits the generalizability of the results and should be kept in mind. Similarly, in the quasi-experimental and longitudinal cohort studies, estimates of joint timing were combined with unknown correlation coefficients. The ad-hoc estimate of $\mathrm{r}=0.50$ is likely to differ between studies and will have affected the analysis. When the true correlations would be larger, these studies' weights will have been larger and shifted the mean and $95 \%$ CI and PI more. Depending on the estimate, this may have either broadened or narrowed the intervals. The absolute values of the $95 \%$ intervals from the meta-analysis should thus only be taken as a preliminary result and warrant further research.

\section{The proximal-to-distal sequence for talent identification}

Aside from the limitations, the results were very consistent in the overall topology of the timing, i.e. in the order in which joint motions occurred and in the differences of timing between consecutive degrees-of-freedom. This provides a starting point for discussions on talent identification based on coordination. Currently, talent identification programs use primarily anthropometric and performance variables (outcome measures) in an attempt to select those athletes with the highest potential of reaching the highest competitive levels. Despite relatively low prognostic validity and a plethora of theoretical, ethical, statistical and technical issues related to talent identification and selection (Baker et al., 2017; Lidor et al., 2005; Matthys et al., 2013; Mohamed et al., 2009; Schorer et al., 2017), their practice in national sports organizations will likely continue and intensify. Therefore, the best possible set of criteria with evidence-based support is necessary. Besides outcome measures of performance (e.g. throwing speed, accuracy), coordination measures of how a given task is executed may inform coaching staff of young players' intrinsic ability to execute a motion in a biomechanically efficient way.

We propose that the topology of the proximal-to-distal sequence of the pelvis, trunk and upper limb may provide a first coordination measure relevant for talent identification purposes. While we agree that coordination 
profiling techniques based on continuous variables, rather than discrete, are more suitable vehicles for this purpose (Glazier, 2017a), to date only limited research has been performed in this area in team handball (Serrien et al., 2016; Wagner et al., 2012b). To spark discussion between movement scientists and stakeholders in sporting organizations and talent identification/development programs, evidencebased measures with scientific consensus are important. The meta-analysis presented in this article provides an overall view on the proximalto-distal sequence, with evidence obtained from experienced and elite players. The $95 \% \mathrm{CI}$ is a too strict quantitative measure for selection. The $95 \%$ PI is better suited for this purpose, but is currently biased (see limitations paragraph). In trunk flexion in the standing throw with run-up, it is additionally biased by the outlier. Future larger studies may take the current meta-analysis as a starting point to cumulate evidence of experienced and elite players to construct a criterion interval.

Qualitatively, plotting a young athlete's throwing patterns against the existing evidence of elite players may help in the evaluation. The time span of the motion is too fast for accurate qualitative visual evaluation; e.g. a coach will not be able to determine whether maximal trunk rotation velocity precedes that of trunk flexion (separated on average by only $30 \mathrm{~ms}$ ). Current developments in motion analysis equipment and software will make large-scale study and evaluation more feasible in coming years. Coaches and talent identification programs that adopt these techniques can qualitatively compare young players with the data from expert players, summarized in this meta-analysis. When athletes exhibit blocked rotation (moving all joints/segments nearly simultaneously) instead of employing a time lag between proximal and distal joints/segments, the plot will show a nearly horizontal line as compared to the ascending patterns in Figure 2. Selected young athletes that have an intrinsic coordination pattern (i.e. before they engage in intensive training of talent development programs) with proximal-to-distal sequencing are perhaps more likely to be able to quickly learn new techniques and sustain less overuse injuries.

Talent selection is only one part of the framework around development towards toplevel sports. Another part is talent development and (technique) training. With regard to the latter, we do not stipulate that athletes should actively be guided towards adopting this theoretical common optimal motion pattern (role model imitation). It is also unclear how athletes could use feedback to change their timing pattern directly given that the events occur within a very limited timespan. This change will likely occur naturally and the optimal pattern is most likely different between and also within players (coordination variability). Rather, talent development programs should strive more towards enabling athletes to explore their own coordination potential through variable and differential training programs (Schöllhorn et al., 2010, 2012); see Wagner and Müller (2008) for a case study of this training method in team handball. Also practices of different movements with proximal-to-distal sequencing patterns may be beneficial for pubescent and adolescent athletes in talent development programs (Wagner et al., 2014). Studies have shown that subjects with expertise in more than one movement with a proximal-to-distal sequence show greater temporal differences between the different motions, indicating that they have found taskspecific optimal patterns (Adrian and Engberg, 1971; Anderson, 1979). We hope that the collective evidence in this review will spark further discussion between scientists and sports practitioners to include coordination measures like the proximal-to-distal sequence into talent selection and that future prospective studies may asses its viability.

\section{Conclusion}

The meta-analysis in this study showed that elite team handball players exhibited a topologically similar proximal-to-distal sequence. For timing of maximal velocities, the sequence was qualitatively the same in three important throwing types, starting with pelvis rotation and followed by trunk rotation, trunk flexion, elbow extension and shoulder internal rotation. The results are discussed in the light of talent detection using this sequence as the coordination profile, but there is still much research necessary before it can be transferred to practice. 


\section{Acknowledgements}

We would like to thank dr. Herbert Wagner and dr. Roland van den Tillaar for sharing their data to perform meta-analysis.

10.1371/journalpcbi.1006013

\section{References}

Adrian MJ, Engberg ML. Sequential timing of three overhead patterns. In: Widule C (Ed.) Kinesiology Review. Washington D.C.: AAPHER, 1-9; 1971

Aguinaldo AL, Buttermore J, Chambers H. Effects of upper trunk rotation on shoulder joint torque among baseball pitchers of various levels. J Appl Biomech, 2007; 23: 42-51

Aguinaldo AL, Chambers $\mathrm{H}$. Correlation of throwing mechanics with elbow valgus load in adult baseball pitchers. Am J Sports Med, 2009; 37: 2043-2048

Anderson MB. Comparison of Muscle Patterning in the Overarm Throw and Tennis Serve. Res Quarterly Am Alliance Heal Phys Educ Recreat Danc, 1979; 50: 541-553

Baeyens JP, Van Roy P, Clarijs JP. Intra-articular kinematics of the normal glenohumeral joint in the late preparatory phase of throwing: Kaltenborn's rule revisited. Ergonomics, 2000; 43: 1726-1737

Baeyens JP, Van Roy P, De Schepper A, Declerq G, Clarijs JP. Glenohumeral joint kinematics related to minor anterior instability of the shoulder at the end of the late preparatory phase of throwing. Clin Biomech, 2001; 16: 752-757

Baker J, Schörer J, Wattie N. Compromising Talent: Issues in Identifying and Selecting Talent in Sport. Quest, 2017; 70: 48-63

Baker J, Young B. 20 Years Later: Deliberate Practice and the Development of Expertise in Sport. Int Rev Sport Exerc Psychol, 2014; 7: 135-157

Bonnefoy-Mazure A, Slawinski J, Riquet A, Lévèque J-M, Miller C, Chèze L. Rotation sequence is an important factor in shoulder kinematics. Application to the elite players' flat serves. J Biomech, 2010; 43: 2022-2025

Borenstein M, Hedges LV, Higgins JPT, Rothstein HR. Introduction to Meta-Analysis. West Sussex: Wiley; 2009

Creveaux T, Sevrez V, Dumas R, Chèze L, Rogowski I. Rotation sequence to report humerothoracic kinematics during 3D motion involving large horizontal component: application to the tennis forehand drive. Sport Biomech, 2018; 17: 131-141

Feltner ME, Dapena J. Three-dimensional interactions in a two-segment kinetic chain. Part I: general model. Int J Sport Biomech, 1989; 5: 403-419

Ferdinands RED, Kersting UG, Marshall RN. Kinematic and kinetic energy analysis of segmental sequencing in cricket fast bowling. Sport Technol, 2013; 6: 10-21

Glazier PS. Could sports biomechanics provide the missing pieces to the talent identification and development puzzle? In: Baker J, Cobley S, Schorer J, Wattie N (Eds.). Routledge Handbook of Talent Identification and Development in Sport. London: Routledge, 236-249; 2017b

Glazier PS. Towards a Grand Unified Theory of sports performance. Hum Mov Sci, 2017a; 56: 139-156

Hirashima M, Kadota H, Sakurai S, Kudo K, Ohtsuki T. Sequential muscle activity and its functional role in the upper extremity and trunk during overarm throwing. J Sports Sci, 2002; 20: 301-310

Jöris H, Edwards van Muyen A, van Ingen Schenau G, Kemper HCG. Force, velocity and energy flow during the overarm throw in female handball players. J Biomech, 1985; 18: 409-414

Knudson D. Significant and meaningful effects in sports biomechanics research. Sport Biomech, 2009; 8: $96-$ 104

Knudson D. Confidence crisis of results in biomechanics research. Sport Biomech, 2017; 16: 425-433

Lidor R, Falk B, Arnon M, Cohen Y, Segal G, Lander Y. Measurement of talent in team handball: the questionable use of motor and physical tests. J Strength Cond Res, 2005; 19: 318-325

Liu H, Leigh S, Yu B. Sequences of upper and lower extremity motions in javelin throwing. J Sports Sci, 2010; 28: 1459-1467

Marshall RN, Elliott BC. Long-axis rotation: The missing link in proximal-to-distal segmental sequencing. J Sports Sci, 2000; 18: 247-254 
Matthys SPJ, Vaeyens R, Fransen J, Deprez D, Pion J, Vandendriessche J, Vandorpe B, Lenoir M, Philippaerts R. A longitudinal study of multidimensional performance characteristics related to physical capacities in youth handball. J Sports Sci, 2013; 31: 325-334

Mohamed H, Vaeyens R, Matthys S, Multael M, Lefevre J, Lenoir M, Philippaerts R. Anthropometric and performance measures for the development of a talent detection and identification model in youth handball. J Sports Sci, 2009; 27: 257-266

Oyama S, Yu B, Blackburn JT, Padua DA, Li L, Myers JB. Improper trunk rotation sequence is associated with increased maximal shoulder external rotation angle and shoulder joint force in high school baseball pitchers. Am J Sports Med, 2014; 42: 2089-2094

Schöllhorn WI, Beckmann H, Davids K. Exploiting system fluctuations. Differential training in physical prevention and rehabilitation programs for health and exercise. Med (Kaunas) 2010; 46: 365-373

Schöllhorn WI, Hegen P, Davids K. The Nonlinear Nature of Learning - A Differential Learning Approach. Open Sports Sci J, 2012; 5: 100-112

Schorer J, Rienhoff R, Fischer L, Baker J. Long-Term Prognostic Validity of Talent Selections: Comparing National and Regional Coaches, Laypersons and Novices. Front Psychol, 2017; 8: 1-8

Serrien B, Clijsen R, Blondeel J, Goossens M, Baeyens JP. Differences in ball speed and three-dimensional kinematics between male and female handball players during a standing throw with run-up. BMC Sports Sci Med Rehabil, 2015; 7, DOI: 10.1186/s13102-015-0021-x

Serrien B, Clijsen R, Anders S, Goossens M, Baeyens JP. Intra-seasonal Variability of Ball Speed and Coordination of Two Team-Handball Throwing Techniques in Elite Male Adolescent Players. Int J Comput Sci Sport, 2016; 15: 1-29

Serrien B, Baeyens JP. The Proximal-to-Distal Sequence in Upper-Limb Motions on Multiple Levels and Time Scales. Hum Mov Sci, 2017; 55: 156-171

van den Tillaar R, Ettema G. Instructions emphasizing velocity, accuracy or both on kinematics of overarm throwing by experienced team handball players. Percept Mot Skills, 2003; 97: 731-742

van den Tillaar R, Ettema G. A comparison between novices and experts of the velocity-accuracy trade-off in overarm throwing. Percept Mot Skills, 2006; 103: 503-514

van den Tillaar R, Ettema G. A three-dimensional analysis of overarm throwing in experienced handball players. J Appl Biomech, 2007; 23: 12-19

van den Tillaar R, Ettema G. A Comparison of Overarm Throwing With the Dominant and Nondominant Arm in Experienced Team Handball Players. Percept Mot Skills, 2009a; 109: 315-326

van den Tillaar R, Ettema G. Is there a proximal-to-distal sequence in overarm throwing in team handball? J Sports Sci, 2009b; 27: 949-955

van den Tillaar R, Ettema G. A comparison of kinematics between overarm throwing with $20 \%$ underweight, regular, and 20\% overweight balls. J Appl Biomech, 2011; 27: 252-257

van den Tillaar R, Marques MC. Effect of training on the kinematics and performance in overarm throwing in experienced female handball players. Port J Sport Sci, 2011; 11: 125-128

van den Tillaar R, Cabri JMH. Gender differences in the kinematics and ball velocity of overarm throwing in elite team handball players. J Sports Sci, 2012; 30: 807-813

van den Tillaar R, Zondag A, Cabri J. Comparing performance and kinematics of throwing with a circular and whip-like wind up by experienced handball players. Scand J Med Sci Sport, 2013; 23: 373-380

Urbin MA, Fleisig GS, Abebe A, Andrews JR. Associations between timing in the baseball pitch and shoulder kinetics, elbow kinetics, and ball speed. Am J Sports Med, 2013; 41: 336-342

Wagner H, Kainrath S, Müller E. Coordinative and tactical parameters in the handball throw and their influence to the level of performance. 13 $3^{\text {th }}$ Annual Congress of the European College of Sport Science, 2008

Wagner H, Müller E. The effects of differential and variable training on the quality parameters of a handball throw. Sport Biomech, 2008; 7: 54-71

Wagner H, Buchecker M, von Duvillard SP, Müller E. Kinematic comparison of team handball throwing with two different arm positions. Int J Sports Physiol Perform, 2010a; 5: 469-483

Wagner H, Buchecker M, von Duvillard SP, Müller E. Kinematic description of elite vs . low level players in team-handball jump throw. J Sport Sci Med, 2010b; 9: 15-23 
Wagner H, Pfusterschmied J, von Duvillard SP, Müller E. Performance and kinematics of various throwing techniques in team-handball. J Sport Sci Med, 2011; 10: 73-80

Wagner H, Pfusterschmied J, von Duvillard SP, Müller E. Skill-dependent proximal-to-distal sequence in team-handball throwing. J Sports Sci, 2012a; 30: 21-29

Wagner H, Pfusterschmied J, Klous M, von Duvillard SP, Müller E. Movement variability and skill level of various throwing techniques. Hum Mov Sci, 2012b; 31: 78-90

Wagner H, Pfusterschmied J, Tilp M, Landlinger J, von Duvillard SP, Müller E. Upper-body kinematics in team-handball throw, tennis serve, and volleyball spike. Scand J Med Sci Sports, 2014a; 24: 345-354

Wagner H, Finkenzeller T, Würth S, von Duvillard SP. Individual and Team Performance in Team-Handball: A Review. J Sport Sci Med, 2014b; 13: 808-816

Wu G, van der Helm FCT, Veeger HEJ, Makhsous M, Van Roy P, Anglin C, Nagels J, Karduna AR, McQuade $\mathrm{K}$, Wang X, Werner FW, Bucholz B. ISB recommendation on definitions of joint coordinate systems of various joints for the reporting of human joint motion-Part II: shoulder, elbow, wrist and hand. $J$ Biomech, 2005; 38: 981-992

Wu G, Siegler S, Allard P, Kirtley C, Leardini A, Rosenbaum D, Whittle M, D'Lima DD, Cristofolini L, Witte $\mathrm{H}$, Schmid O, Stokes I. ISB recommendation on definitions of joint coordinate system of various joints for the reporing of human joint motion - part I: ankle, hip and spine. J Biomech, 2002; 35: 543-548

Zhang Z, Guo D, Huber ME, Park SW, Sternad D. Exploiting the geometry of the solution space to reduce sensitivity to neuromotor noise. Plos Comput Biol 2018; 14, DOI: 10.1371/journalpcbi.1006013

\section{Corresponding author:}

\section{Ben Serrien}

Vrije Universiteit Brussel - Faculty of Physical Education and Physiotherapy

Pleinlaan 2

1050 Brussels (BELGIUM)

Tel: 0032489406776Address:

E-mail: ben.serrien@vub.be 